\title{
[2+2] Cycloaddition Reactions with a Tungsten-Stabilized 2H-Phenol
}

Michael A. Todd, Michal Sabat, William H. Myers\#, W. Dean Harman*

Contribution from the Department of Chemistry, University of Virginia Charlottesville, VA 229044319

\# Department of Chemistry, University of Richmond, Richmond, Virginia 23173 wdh5z@virginia.edu

\section{Supplementary Material}

Contents:

Experimental Details.............................S2-S6

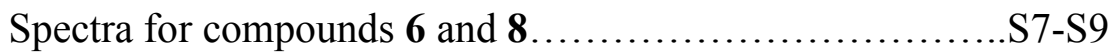




\section{Experimental Details.}

General Methods. All NMR spectra were obtained on either a 300 or $500 \mathrm{MHz}$ Varian INOVA spectrometer, or on a 300 or $500 \mathrm{MHz}$ Bruker Avance spectrometer. All chemical shifts are reported in ppm versus tetramethylsilane using residual shifts of the deuterated solvent as the internal standard. All coupling constants $(\mathrm{J})$ are reported in hertz $(\mathrm{Hz})$. All ${ }^{31} \mathrm{P}$ NMR data is reported versus an external standard in acetone (Trimethylphosphate, $\delta$-16.58 ppm). Infrared spectra were obtained on a MIDAC Prospect Series spectrometer as a glaze on a Horizontal Attenuated Total Reflectance (HATR) cell from Pike Industries. Electrochemical measurements were taken under a nitrogen atmosphere using a BAS Epsilon EC-2000 potentiostat. Cyclic voltammetry data were obtained in a three electrode cell from +1.7 to $-1.7 \mathrm{~V}$, with a glassy carbon working electrode, a platinum wire auxiliary electrode and a platinum wire reference electrode. All data were obtained using a $100 \mathrm{mv} / \mathrm{s}$ scan rate with tetrabutylammonium hexafluorphosphate (TBAH) as the electrolyte in N,N-dimethylacetamide (DMA) unless otherwise noted. All potentials were reported versus the Normal Hydrogen Electrode (NHE) using cobaltocenium hexafluorophosphate $\left(\mathrm{E}_{1 / 2}=-0.78 \mathrm{~V}\right)$ as an internal standard. For reversible waves the peak to peak separation was less than $100 \mathrm{mV}$. All HRMS data was obtained using a 1:1 water:acteonitrile solution with sodium trifluoroacetate as a standard. The data for metal complexes is reported using the five most intense peaks from the isotopic envelope, and is listed as $\mathrm{m} / \mathrm{z}$ with the intensity relative to the most abundant peak of the isotopic envelope given in parentheses for both the calculated and observed peaks. The difference between calculated and observed peaks is reported in ppm. For isolated organic products, the monoisotopic peak is reported, with observed, calculated, and ppm difference again provided. Thin layer chromatography was performed on a Uniplate silica gel GF from Analtech Inc. Methylene chloride and benzene were all run down a column packed with activated alumina and purged with nitrogen prior to use. All other solvents and chemicals were used as received from Sigma-Aldrich, Acros Chemicals or Fischer Scientific. Compound 1 was previously reported. ${ }^{1}$

\section{$\operatorname{TpW}(\mathrm{NO})\left(\mathrm{PMe}_{3}\right)\left(\eta^{2}-\right.$ phenol) (1b)}

In an Erlenmeyer flask with a ground glass joint $40 \mathrm{~mL}$ methanol was purged for 10 min with argon. TpW(NO) $\left(\mathrm{PMe}_{3}\right)\left(\eta^{2}\right.$-phenol) (1a,b) $(4.068 \mathrm{~g}, 6.814 \mathrm{mmol})$ and a stir bar were added. The reaction was purged for $5 \mathrm{~min}$ and DBU $(0.249 \mathrm{~g}, 1.596 \mathrm{mmol})$ was added. The flask was sealed with a glass stopper. After $3 \mathrm{~d}$ the reaction was filtered and washed with $2 \mathrm{~mL}$ methanol and dried in vacuo. A light yellow precipitate was collected 1b (3.298g, 81\% yield.) Since ketenes react with methanol the solid was reprecipitated using methylene chloride $(10 \mathrm{~mL})$ and hexanes $(400 \mathrm{~mL})$ to remove any remaining methanol prior to the synthesis of $\mathbf{2 a}, \mathbf{b}, \mathbf{3 a}, \mathbf{b}, \mathbf{4 a}, \mathbf{b}$ and $\mathbf{5}$.

\section{TpW(NO) $\left(\mathrm{PMe}_{3}\right)\left(\eta^{2}\right.$-4,5-(7-chlorobicyclo[4.2.0]oct-4-ene-3,8-dione)) (2a,b)}

In a glovebox under a dinitrogen atmosphere, using flame dried glassware, a solution of chloroacetylchloride $(0.140 \mathrm{~g}, 1.24 \mathrm{mmol})$ in $75 \mathrm{~mL} \mathrm{CH}_{2} \mathrm{Cl}_{2}$ was slowly added drop-wise to a well stirred solution of $\mathbf{1 b}(0.500 \mathrm{~g}, 0.838 \mathrm{mmol})$ and DIEA $(0.167 \mathrm{~g}, 1.29$ 
mmol) in $25 \mathrm{~mL} \mathrm{CH}_{2} \mathrm{Cl}_{2}$. After about 1 hour the addition was complete and the reaction was removed from the glovebox. $\mathrm{HCl}_{(\mathrm{aq})}(1 \mathrm{M}, 100 \mathrm{~mL})$ was added and the $\mathrm{CH}_{2} \mathrm{Cl}_{2}$ layer was extracted, dried with $\mathrm{MgSO}_{4}$, filtered and evaporated by rotatory evaporation. The residue was dissolved in $1 \mathrm{~mL} \mathrm{CH} \mathrm{Cl}_{2}$. Diethyl ether $(10 \mathrm{~mL})$ was slowly added and the resulting precipitate was filtered, washed with five $10 \mathrm{~mL}$ portions of ether and dried in vacuo. The light beige precipitate was isolated as a mixture of two diastereomers $(\mathbf{2 a}, \mathbf{b}$, 1.4:1 dr, 0.517g, $92 \%)$. ${ }^{1} \mathrm{H}$ NMR $\left(\mathrm{CDCl}_{3}\right): \delta 1.12\left(\mathrm{~d}, 9 \mathrm{H}, \mathrm{J}=8.4 \mathrm{~Hz}, \mathrm{PMe}_{3} \mathrm{~A}\right), 1.17(\mathrm{~d}$, 9H, J=8.7 Hz, PMe 3 B), 2.01 (d, 1H, J=9.6 Hz, H4 A), 2.18 (d, 1H, J=9.6 Hz, H4 B), 2.58 (d, 1H, J=18.0, H2 B), 2.64 (d, 1H, J=16.8, H2 A), 2.87 (m, 2H, H2 A and B), 3.26 (dd, 1H, J=13.2, 9.6 Hz, H5 A), 3.35 (dd, 1H, J=9.3, 6.9 Hz, H6 B), 3.46 (dd, 1H, J=12.6, 9.6 Hz, H5 B), 3.69 (br t, J=9.3 Hz, H1 A), 3.87 (m, 2H, H6 A, H1 B) 4.77 (dd, J=6.9, 3.0 Hz, H7 B), 5.22 (dd, J=9.0, 2.1 Hz, H7 A), 6.09 (t, 1H, J=2.4 Hz, Tp), 6.11 (t, 1H, J=2.4 $\mathrm{Hz}, \mathrm{Tp}), 6.22$ (t, 1H, J=2.4 Hz, Tp), 6.24 (t, 1H, J=2.4 Hz, Tp), 6.34 (t, 1H, J=2.4 Hz, Tp), 6.35 (t, 1H, J=2.4 Hz, Tp), 7.31 (d, 1H, J=2.4 Hz, Tp), 7.36 (d, 1H, J=2.4 Hz, Tp), 7.42 (d, 1H, J=2.4 Hz, Tp), 7.45 (d, 1H, J=2.4 Hz, Tp), 7.54 (d, 1H, J=2.4 Hz, Tp), 7.68 (d, 1H, J=2.4 Hz, Tp), 7.70 (d, 1H, J=2.4 Hz, Tp), 7.76 (d, 1H, J=2.4 Hz, Tp), 8.09 (d, $1 \mathrm{H}, \mathrm{J}=2.4 \mathrm{~Hz}, \mathrm{Tp}) .{ }^{13} \mathrm{C} \mathrm{NMR}\left(\mathrm{CDCl}_{3}\right): \delta 12.5\left(\mathrm{~d}, \mathrm{~J}=32 \mathrm{~Hz}, \mathrm{PMe}_{3} \mathrm{~A}\right), 12.9(\mathrm{~d}, \mathrm{~J}=30 \mathrm{~Hz}$, $\mathrm{PMe}_{3} \mathrm{~B}$ ), 31.8 (s, C2 B), 32.3 (s, C2 A), 32.9 (d, J=4.0 Hz, C6 A) 41.4 (d, J=3.0 Hz, C6 B), 54.7(s, C1 B), 55.1 (s, C1 A), 57.3 (d, J=12 Hz, C5 A), 59.4 (s, C4 B), 60.6 (s, C4 A), 65.1 (d, J=13, C5 B), 68.5 (s, C7 A), 73.1 (s, C7 B), 106.1 (s, Tp), 106.2 (s, Tp), 106.8 (s, Tp), 106.9 (s, Tp), 107.3 (s, Tp), 107.4 (s, Tp), 136.3 (s, Tp), 136.4 (s, Tp), 136.9 (s, Tp), 137.2 (s, 2Tp), 139.9 (s, Tp), 140.0 (s, Tp), 142.7 (s, Tp), 143.1 (s, 2Tp), 143.6 (s, 2Tp), $202.0(\mathrm{~s}, \mathrm{C} 8 \mathrm{~A}), 203.8(\mathrm{~s}, \mathrm{C} 3 \mathrm{~B}), 204.2(\mathrm{~s}, \mathrm{C} 8 \mathrm{~A}), 205.7(\mathrm{~s}, \mathrm{C} 3 \mathrm{~B}) .{ }^{31} \mathrm{P}$ NMR $\left(\mathrm{CDCl}_{3}\right): \delta$ 8.0 (A), -8.8 (B). IR: $v_{\mathrm{NO}}=1567, v_{\mathrm{CO}}=1621, v_{\mathrm{CO}}=1787 \mathrm{~cm}^{-1} . \mathrm{CV}: \mathrm{E}_{\mathrm{p}, \mathrm{a}}=+1.14 \mathrm{~V}$. Anal Calc'd for $\mathrm{C}_{20} \mathrm{H}_{26} \mathrm{BClN} 7 \mathrm{O}_{3} \mathrm{PW} \cdot \mathrm{CH}_{2} \mathrm{Cl}_{2}$ : C, 33.25; H, 3.72; N, 12.93. Found: $\mathrm{C}, 33.72 ; \mathrm{H}$, 3.78; N, 13.00. HRMS:[M + H] obs'd (\%), calc'd (\%), diff. in ppm: 672.11771 (65.9), 672.11807 (67.1), -0.5; 673.11983 (62.5), 673.12046 (67.0), -0.9; 674.11835 (100.0), 674.11931 (100.0), -1.4; 675.12128 (49.3), 675.12207 (52.8), -1.2; 676.12037 (89.6), $676.12200(91.4),-2.4$.

\section{TpW(NO) $\left(\mathrm{PMe}_{3}\right)\left(\eta^{2}\right.$-4,5-(7-(3-methoxyphenyl)bicyclo[4.2.0]oct-4-ene-3,8-dione)) (3a,b)}

In a glovebox under a dinitrogen atmosphere, using flame dried glassware, a solution of oxalyl chloride $(1.608 \mathrm{~g}, 12.66 \mathrm{mmol})$ in $1 \mathrm{~mL}$ benzene was added to 3 methoxyphenylacetic acid $(0.128 \mathrm{~g}, 0.771 \mathrm{mmol})$. After $4 \mathrm{~h}$ the solvent was removed via evacuation. $1 \mathrm{~mL}$ benzene was added, evacuated to remove any remaining oxalyl chloride. The resulting oil was dissolved in $20 \mathrm{~mL} \mathrm{CH}_{2} \mathrm{Cl}_{2}$ and slowly added dropwise to a well stirred solution of $1 \mathbf{b}(0.199 \mathrm{~g}, 0.333 \mathrm{mmol})$ and DIEA $(0.101 \mathrm{~g}, 0.783 \mathrm{mmol})$ in 10 $\mathrm{mL} \mathrm{CH} \mathrm{Cl}_{2}$. After about 30 minutes the addition was complete and the reaction was removed from the glovebox. $\mathrm{HCl}_{(\mathrm{aq})}(1 \mathrm{M}, 40 \mathrm{~mL})$ was added and the $\mathrm{CH}_{2} \mathrm{Cl}_{2}$ layer was extracted, dried with $\mathrm{MgSO}_{4}$, filtered and evaporated using rotatory evaporation. Acetone $(15 \mathrm{~mL})$ was added to the residue and the mixture was vigorously stirred. After 15 minutes the reaction was filtered and washed with $1 \mathrm{~mL}$ acetone, yielding an off-white solid (3a, $0.072 \mathrm{~g}, 29$ \%yield). ${ }^{1} \mathrm{H}$ NMR $\left(\mathrm{CDCl}_{3}\right): \delta 1.09$ (d, 9H, J=8.7 Hz, $\left.\mathrm{PMe}_{3}\right), 2.26$ (d, 1H, J=9.3 Hz, H4), 2.72 (d, 1H, J=18.0 Hz, H2), 2.98 ( dd, 1H, J=18.0, $12.0 \mathrm{~Hz}, \mathrm{H} 2$ ), 
3.51 (m, 2H, H6, H5), 3.80 (m buried, H1), 3.80 (s, 3H, OMe), 4.41 (dd, 1H, J=7.2, 2.4 $\mathrm{Hz}, \mathrm{H} 7), 6.16$ (d, 1H, J=2.4 Hz, Tp), 6.23 (d, 1H, J=2.4 Hz, Tp), 6.37 (d, 1H, J=2.4 Hz, Tp), 6.78-6.88 (m, 3H), $7.27(\mathrm{~m}, 1 \mathrm{H}), 7.33(\mathrm{t}, 1 \mathrm{H}, \mathrm{J}=2.4 \mathrm{~Hz}, \mathrm{Tp}), 7.53(\mathrm{t}, 1 \mathrm{H}, \mathrm{J}=2.4 \mathrm{~Hz}$, $\mathrm{Tp}), 7.56$ (t, 1H, J=2.4 Hz, Tp), 7.70 (t, 1H, J=2.4 Hz, Tp), 7.77 (t, 1H, J=2.4 Hz, Tp), $8.13(\mathrm{t}, 1 \mathrm{H}, \mathrm{J}=2.4 \mathrm{~Hz}, \mathrm{Tp}) .{ }^{3} \mathrm{C}$ NMR $\left(\mathrm{CDCl}_{3}\right): \delta 12.7$ (d, J=29 Hz), $32.7(\mathrm{~s}), 37.8(\mathrm{~s})$, $55.5(\mathrm{~s}), 56.5(\mathrm{~s}), 60.2(\mathrm{~s}), 67.9(\mathrm{~d}, \mathrm{~J}=13 \mathrm{~Hz}), 78.3(\mathrm{~s}), 106.4(\mathrm{~s}), 106.7(\mathrm{~s}), 107.4(\mathrm{~s})$, $112.6(\mathrm{~s}), 113.3(\mathrm{~s}), 119.6(\mathrm{~s}), 130.2(\mathrm{~s}), 136.2(\mathrm{~s}), 137.0(\mathrm{~s}), 137.1(\mathrm{~s}), 138.5(\mathrm{~s}), 140.0$ $(\mathrm{s}), 143.0(\mathrm{~s}), 143.6(\mathrm{~s}), 160.2(\mathrm{~s}), 206.7(\mathrm{~s}), 210.8(\mathrm{~s})$.

The filtrate was added to $50 \mathrm{~mL}$ hexane and the resulting precipitate was collected by filtration (3b, 0.128g, 52\% yield.) ${ }^{1} \mathrm{H} \mathrm{NMR}\left(\mathrm{CDCl}_{3}\right): \delta 0.97$ (d, 9H, J=8.4 Hz, $\left.\mathrm{PMe}_{3}\right)$, $2.04(\mathrm{~d}, 1 \mathrm{H}, \mathrm{J}=9.6 \mathrm{~Hz}, \mathrm{H} 4), 2.66$ (dd, 1H, J=13.8, 9.6 Hz, H5), 2.80 (d, 1H, J=17.1 Hz, H2), 2.98 (dd, 1H, J=17.1, 7.5 Hz, H2), 3.72 (s, 3H, OMe), 3.92 (m, 2H, H1, H6), 4.90 (dd, 1H, J=9.0, 3.0 Hz, H7), 6.11 (t, 1H, J=2.1 Hz, Tp), 6.14 (t, 1H, J=2.1 Hz, Tp), 6.35 (t, 1H, J=2.1 Hz, Tp), 6.65 (d, 1H, J=2.1 Hz, Tp), 6.83-6.92 (m, 3H), $7.24(\mathrm{~m}, 1 \mathrm{H}), 7.52$ $(\mathrm{d}, 1 \mathrm{H}, \mathrm{J}=2.1 \mathrm{~Hz}, \mathrm{Tp}), 7.53(\mathrm{~d}, 1 \mathrm{H}, \mathrm{J}=2.1 \mathrm{~Hz}, \mathrm{Tp}), 7.62(\mathrm{~d}, 1 \mathrm{H}, \mathrm{J}=2.1 \mathrm{~Hz}, \mathrm{Tp}), 7.74(\mathrm{~d}$, $1 \mathrm{H}, \mathrm{J}=2.1 \mathrm{~Hz}, \mathrm{Tp}), 8.11(\mathrm{~d}, 1 \mathrm{H}, \mathrm{J}=2.1 \mathrm{~Hz}, \mathrm{Tp}) .{ }^{13} \mathrm{C} \mathrm{NMR}\left(\mathrm{CDCl}_{3}\right): \delta 12.7$ (d, J=29 Hz, $\mathrm{PMe}_{3}$ ), 32.9 (s, C2), 33.3 (d, J=4 Hz, C6), 55.4 (s, OMe), 57.1 (s, C1), 61.2 (s, C4), 61.5 (d, J=12.0, C5), 71.4 (s, C7), 106.0 (s,Tp), 106.7 (s,Tp), 107.3 (s,Tp), 114.2 (s. Ph), 114.8 (s, Ph), 122.6 (s, Ph), 129.4 (s, Ph), 135.2 (s, Ph), 136.2 (s,Tp), 136.8 (s,Tp), 137.1 (s,Tp), 139.2 (s,Tp), 143.1 (s,Tp), 143.6 (s,Tp), 159.5 (s, Ph), 205.4 (s, C3), 210.1 (s, C8). IR: $v_{\mathrm{NO}}=1565, v_{\mathrm{CO}}=1600, v_{\mathrm{CO}}=1766 \mathrm{~cm}^{-1} . \mathrm{CV}: \mathrm{E}_{\mathrm{p}, \mathrm{a}}=+0.94 \mathrm{~V}$. Anal Calc'd for $\mathrm{C}_{27} \mathrm{H}_{33} \mathrm{BN}_{7} \mathrm{O}_{4} \mathrm{PW} \cdot \mathrm{CH}_{2} \mathrm{Cl}_{2}: \mathrm{C}, 40.51 ; \mathrm{H}, 4.25 ; \mathrm{N}, 11.91$. Found: $\mathrm{C}, 40.82 ; \mathrm{H}, 4.25 ; \mathrm{N}$, 11.81 .

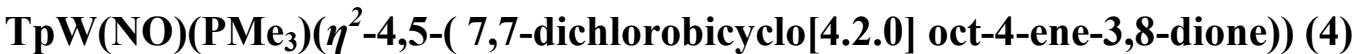

Diisopropylethylamine $\left(0.043 \mathrm{~g}, 0.34 \mathrm{mmol}\right.$ ) was dissolved in $10 \mathrm{~mL} \mathrm{CH}_{2} \mathrm{Cl}_{2}$, added to $\mathbf{1 b}(0.100 \mathrm{~g}, 0.168 \mathrm{mmol})$ and stirred. Dichloroacetylchloride $(0.049 \mathrm{~g}, 0.34$ mmol) was dissolved in $10 \mathrm{~mL} \mathrm{CH}_{2} \mathrm{Cl}_{2}$ and slowly added dropwise via a separatory funnel over about 20 minutes. The reaction was removed from the glovebox and $\mathrm{HCl}_{(\mathrm{aq})}$ $(1 \mathrm{M}, 20 \mathrm{~mL})$ was added. The reaction was extracted, dried with $\mathrm{MgSO}_{4}$ and evaporated using rotatory evaporation. $2 \mathrm{~mL}$ EtOAc was added and the reaction was stirred. After 5 minutes the reaction was filtered, washed twice with $1 \mathrm{~mL}$ EtOAc, dried in vacuo. An light beige precipitate was isolated $\left(4,0.077 \mathrm{~g}, 65 \%\right.$ yield). ${ }^{1} \mathrm{H} \mathrm{NMR}\left(\mathrm{CDCl}_{3}\right): \delta 1.18$ (d, 9H, J=8.4 Hz, $\mathrm{PMe}_{3}$ ), 2.18 (d, 1H, J=9.6 Hz, H4), 2.74 (d, 1H, J=17.5 Hz, H2), 2.89 (dd, $1 \mathrm{H}, \mathrm{J}=17.5,10.4 \mathrm{~Hz}, \mathrm{H} 2$ ), 3.34 (dd, 1H, J=12.9, $9.6 \mathrm{~Hz}, \mathrm{H} 5), 3.95$ (d, 1H, J=10.4 Hz, H6), 4.27 (t, 1H, J=10.4 Hz, H1), 6.14 (t, J=2.1 Hz, Tp), 6.28 (t, J=2.1 Hz, Tp), 6.39 (t, $\mathrm{J}=2.1 \mathrm{~Hz}, \mathrm{Tp}), 7.37$ (d, J=2.1 Hz, Tp), 7.43 (d, J=2.1 Hz, Tp), 7.56 (d, J=2.1 Hz, Tp), 7.72 (d, J=2.1 Hz, Tp), 7.79 (d, J=2.1 Hz, Tp), 8.13 (d, J=2.1 Hz, Tp). ${ }^{13} \mathrm{C} \mathrm{NMR}\left(\mathrm{CDCl}_{3}\right)$ : $\delta 12.6$ (d, J=29 Hz), 32.0 (s), 49.3 (d, J=4 Hz), 54.9 (s), 59.5 (d, J=12 Hz), 60.1(s), 93.3(s), 106.3(s), 107.0 (s), 107.5 (s), 136.5 (s), 137.1 (s), 137.3 (s), 139.9 (s), 143.2 (s), $143.7(\mathrm{~s}), 197.1(\mathrm{~s}), 203.8(\mathrm{~s}) .{ }^{31} \mathrm{P} \mathrm{NMR}\left(\mathrm{CDCl}_{3}\right): \delta-10.1$. IR: $\mathrm{v}_{\mathrm{NO}}=1563, \mathrm{v}_{\mathrm{CO}}=1621$, $v_{\mathrm{CO}}=1798 \mathrm{~cm}^{-1} . \mathrm{CV}: \mathrm{E}_{\mathrm{p}, \mathrm{a}}=+1.18 \mathrm{~V}$. Anal Calc'd for $\mathrm{C}_{20} \mathrm{H}_{25} \mathrm{BCl}_{2} \mathrm{~N}_{7} \mathrm{O}_{3} \mathrm{PW}: \mathrm{C}, 33.93 ; \mathrm{H}$, 3.56; N, 13.85. Found: C, 33.65; H, 3.61; N, 13.36 . 
TpW(NO)(PMe 3$)\left(\eta^{2}-4,5-(\right.$ 8-hydroxy-7-(3-methoxyphenyl)bicyclo[4.2.0] oct-4-ene-3one)) (5)

A solution of $3 \mathbf{a}(0.050 \mathrm{~g}, 0.067 \mathrm{mmol})$ in $0.5 \mathrm{~mL}$ of $1: 1 \mathrm{CH}_{2} \mathrm{Cl}_{2}: \mathrm{MeOH}$ was added to $\mathrm{NaBH}_{4}$. After 5 minutes $1 \mathrm{~mL} \mathrm{H}$ O was added. The $\mathrm{CH}_{2} \mathrm{Cl}_{2}$ layer was extracted, dried over $\mathrm{MgSO}_{4}$ and evaporated by rotatory evaporation. The residue was dissolved in $0.25 \mathrm{~mL} \mathrm{CH}_{2} \mathrm{Cl}_{2}$, precipitated into $50 \mathrm{~mL}$ hexanes, filtered and dried in vacuo. The precipitate was isolated as an off-white solid $(5,0.33 \mathrm{~g}, 66 \%)$. ${ }^{1} \mathrm{H}$ NMR $\left(\mathrm{CDCl}_{3}\right): \delta 0.87\left(\mathrm{~d}, 9 \mathrm{H}, \mathrm{J}=8.1 \mathrm{~Hz}, \mathrm{PMe}_{3}\right), 2.23(\mathrm{~d}, 1 \mathrm{H}, \mathrm{J}=9.6 \mathrm{~Hz}, \mathrm{H} 4), 2.41$ (d, 1H, J=2.7 $\mathrm{Hz}, \mathrm{OH}), 2.50$ (d, 1H, J=17.1 Hz, H2), 3.00 (dd, 1H, J=17.1, 7.8 Hz, H2), 3.09 (dd, 1H, $\mathrm{J}=14.1,9.6 \mathrm{~Hz}, \mathrm{H} 5), 3.27$ (q, 1H, J= 7.8 Hz, H1), 3.74 (s, OMe), 3.93 (m, 2H, H6, H7), $4.79(\mathrm{~m}, 1 \mathrm{H}, \mathrm{H} 8), 6.14(\mathrm{~m}, 2 \mathrm{H}), 6.32(\mathrm{t}, 1 \mathrm{H}, \mathrm{J}=2.1 \mathrm{~Hz}), 6.83(\mathrm{~m}, 1 \mathrm{H}), 6.91(\mathrm{~d}, 1 \mathrm{H}, \mathrm{J}=2.1$ $\mathrm{Hz}), 7.18(\mathrm{~m}, 1 \mathrm{H}), 7.52(\mathrm{~m}, 2 \mathrm{H}), 7.62(\mathrm{~m}, 2 \mathrm{H}), 7.72(\mathrm{~d}, \mathrm{~J}=2.1 \mathrm{~Hz}), 8.08(\mathrm{~d}, \mathrm{~J}=2.1 \mathrm{~Hz}) .{ }^{13} \mathrm{C}$ NMR $\left(\mathrm{CDCl}_{3}\right): \delta 12.7$ (d, J=29 Hz, PMe $), 33.5$ (s, C2), 39.6 (s, C3), 39.8 (d, J=4 Hz, C4), 51.7 (s, C8), 55.4 (s, OMe), 61.6 (s, C6), 63.9 (d, J=11 Hz, C5), 73.5 (s, C7), 106.2 (s,Tp), 106.6 (s, Tp), 107.1 (s,Tp), 113.7 (s), 116.3 (s), 124.0 (s), 128.8 (s, 2C), 135.9 (s), $136.6(\mathrm{~s}), 136.8(\mathrm{~s}), 143.6(\mathrm{~s}, 3 \mathrm{C}), 159.0(\mathrm{~s}), 210.6(\mathrm{~s}) . \mathrm{IR}: \mathrm{v}_{\mathrm{NO}}=1563, \mathrm{v}_{\mathrm{CO}}=1595, v_{\mathrm{OH}}=$ $3498 \mathrm{~cm}^{-1}$. CV: $\mathrm{E}_{\mathrm{p}, \mathrm{a}}=+0.74 \mathrm{~V}$. Anal Calc'd for $\mathrm{C}_{27} \mathrm{H}_{35} \mathrm{BN}_{7} \mathrm{O}_{4} \mathrm{PW}: \mathrm{C}, 43.40 ; \mathrm{H}, 4.72 ; \mathrm{N}$, 13.12. Found: $\mathrm{C}, 43.02 ; \mathrm{H}, 4.66 ; \mathrm{N}, 12.83$.

\section{8-hydroxy-7-(3-methoxyphenyl)bicyclo[4.2.0] oct-4-ene-3-one (6)}

A solution of $\mathrm{N}$-Bromosuccinimide $(0.019 \mathrm{~g}, 0.11 \mathrm{mmol})$ in $0.5 \mathrm{~mL} \mathrm{CH}{ }_{3} \mathrm{CN}$ and $0.5 \mathrm{~mL}$ $\mathrm{CH}_{2} \mathrm{Cl}_{2}$ was added to added to $6(0.040 \mathrm{~g}, 0.054 \mathrm{mmol})$. After 10 minutes the solvent was removed by rotatory evaporation. The reaction was chromatographed using a $20 \times 20$, $500 \mu \mathrm{M}$ preparatory TLC plate using 1:1 EtOAc:hexanes as the eluent. Compound 7 $\left(0.0063 \mathrm{~g}, 48 \%, \mathrm{R}_{\mathrm{f}}=0.24\right)$ was isolated as a colorless oil. The recovery of the metal complex was not attempted. ${ }^{1} \mathrm{H}$ NMR $\left(\mathrm{CDCl}_{3}\right)$ : $\delta 1.95$ (br s, $\left.1 \mathrm{H}\right), 2.49$ (dd, $1 \mathrm{H}, \mathrm{J}=18.0$, $7.8 \mathrm{~Hz}), 2.64(\mathrm{dd}, 1 \mathrm{H}, \mathrm{J}=18.0,1.8 \mathrm{~Hz}), 3.25(\mathrm{q}, 1 \mathrm{H}, \mathrm{J}=6.3 \mathrm{~Hz}), 3.49(\mathrm{~m}, 1 \mathrm{H}), 3.79(\mathrm{~s}, 3 \mathrm{H})$, $4.00(\mathrm{dd}, 1 \mathrm{H}, \mathrm{J}=9.3,5.7 \mathrm{~Hz}), 4.71(\mathrm{td}, 1 \mathrm{H}, \mathrm{J}=5.7,2.7 \mathrm{~Hz}), 6.18(\mathrm{dd}, 1 \mathrm{H}, \mathrm{J}=10.5,2.1 \mathrm{~Hz})$, 6.80-6.95 (m, 4H), $7.24(\mathrm{t}, 1 \mathrm{H}, \mathrm{J}=8.1 \mathrm{~Hz}) .{ }^{13} \mathrm{C} \mathrm{NMR}\left(\mathrm{CDCl}_{3}\right): \delta 33.8(\mathrm{~s}), 35.1(\mathrm{~s}), 38.4(\mathrm{~s})$, 49.5 (s), 55.4 (s), 74.7 (s), 112.5 (s), 115.7 (s), 122.1 (s), 129.6 (s), 130.5 (s), 137.3 (s), 149.7 (s), $159.8(\mathrm{~s}), 198.4$ (s). IR: $v_{\mathrm{CO}}=1662, \mathrm{v}_{\mathrm{OH}}=3415 \mathrm{~cm}^{-1}$. HRMS: $\left[\mathrm{C}_{15} \mathrm{H}_{16} \mathrm{O}_{3}+\mathrm{Na}^{+}\right]$ obs'd (\%), calc'd (\%), diff. in ppm: 267.09938 (100), 267.09917 (100), 0.8.

\section{TpW(NO)(PMe 3$)\left(\eta^{2}-4,5-(3-0 x o b i c y c l o[4.1 .0]\right.$ hept-4-ene-7-carboxylic acid methyl ester)) (7)}

A mixture of $\mathrm{LiOMe}(0.005 \mathrm{~g}, 0.13 \mathrm{mmol})$ in $0.25 \mathrm{~mL}$ methanol was added to a solution of $\mathbf{2 a}, \mathbf{b}(0.050 \mathrm{~g}, 0.074 \mathrm{mmol})$ in $0.25 \mathrm{~mL} \mathrm{CH}_{2} \mathrm{Cl}_{2}$. After 3 hours the solvent was removed by rotatory evaporation. The residue was dissolved in $1 \mathrm{~mL} \mathrm{CH}_{2} \mathrm{Cl}_{2}$ and extracted with 3 $\mathrm{mL}$ water. The organic layer was dried with $\mathrm{MgSO}_{4}$, filtered and removed by rotatory evaporation. The residue was dissolved in $0.25 \mathrm{~mL}$ methylene chloride, then $2 \mathrm{~mL} \mathrm{Et}_{2} \mathrm{O}$ and $4 \mathrm{~mL}$ hexanes were added. The reaction was filtered and dried in vacuo. An offwhite precipitate $(7,0.029 \mathrm{~g}, 58 \%$ yield $)$ was isolated. ${ }^{1} \mathrm{H} \mathrm{NMR}\left(\mathrm{CDCl}_{3}\right): \delta 1.21(\mathrm{~d}, 9 \mathrm{H}$, $\left.\mathrm{J}=8.7 \mathrm{~Hz}, \mathrm{PMe}_{3}\right), 1.65$ (dd, 1H, J=4.5, 3.6 Hz, H7), 1.97 (d, 1H, J=9.6 Hz, H4), 2.08 (m, 
1H, H1), 2.21 (dd, 1H, J=9.3, 3.6 Hz, H6), 2.68 (d, 1H, J=18.0 Hz, H2), 3.01 (dd, 1H, $\mathrm{J}=18.0,5.4 \mathrm{~Hz}, \mathrm{H} 2), 3.47$ (dd, 1H, J=12.0, $9.6 \mathrm{~Hz}, \mathrm{H} 5), 3.68$ (s, 3H, OMe), 6.12 (t, 1H, $\mathrm{J}=2.4 \mathrm{~Hz}, \mathrm{Tp}), 6.21$ (t, 1H, J=2.4 Hz, Tp), 6.36 (t, 1H, J=2.4 Hz, Tp), 7.28 (d, 1H, J=2.4 Hz, Tp), 7.53 (d, 2H, J=2.4 Hz, Tp), 7.68 (d, 1H, J=2.4 Hz, Tp), 7.75 (d, 1H, J=2.4 Hz, $\mathrm{Tp}), 8.15(\mathrm{~d}, 1 \mathrm{H}, \mathrm{J}=2.4 \mathrm{~Hz}, \mathrm{Tp}) .{ }^{13} \mathrm{C} \mathrm{NMR}\left(\mathrm{CDCl}_{3}\right): \delta 13.1$ (d, J=29 Hz, PMe $), 24.7$ (s, C1), 29.6 (d, J=3 Hz, C6), 31.9 (s, C7), 35.4 (c, C2), 51.7 (s, Me), 60.2 (s, C4), 63.9 (s, C5), 106.1 (s, Tp), 106.5 (s, Tp), 107.3 (s, Tp), 136.0 (s, Tp), 140.0 (s, Tp), 143.0 (s, Tp),143.8 (s, Tp), 174.7 (s, C8), 207.2 (s, C3). IR: $v_{\mathrm{NO}}=1571, v_{\mathrm{CO}}=1598 v_{\mathrm{CO}}=$ $1712 \mathrm{~cm}^{-1}$. CV: $\mathrm{E}_{\mathrm{p}, \mathrm{a}}=+0.86 \mathrm{~V}$. Anal Calc'd for $\mathrm{C}_{21} \mathrm{H}_{29} \mathrm{BN}_{7} \mathrm{O}_{4} \mathrm{PW}: \mathrm{C}, 37.69 ; \mathrm{H}, 4.37 ; \mathrm{N}$, 14.65. Found: C, 37.34; H, 4.49; N, 14.23.

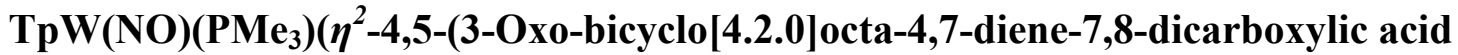 dimethyl ester)) (8)}

Dimethyl acetylenedicarboxylate $(0.146 \mathrm{~g}, 1.03 \mathrm{mmol})$ was dissolved in $0.25 \mathrm{~mL}$ $\mathrm{CH}_{2} \mathrm{Cl}_{2}$ and added to $\mathbf{1 b}(0.050 \mathrm{~g}, 0.084 \mathrm{mmol})$ and heated at $50^{\circ} \mathrm{C}$. After $6.5 \mathrm{~h}$ the solvent was removed by applying a $\mathrm{N}_{2}$ stream under vacuum. When the residue was completely dry $0.25 \mathrm{~mL} \mathrm{CH} \mathrm{Cl}_{2}$ was added and this soltuion was added to $50 \mathrm{~mL}$ hexanes. The yellow precipitate was collected by filtration and dried in vacuo. The precipitate $(0.057 \mathrm{~g})$ contained mostly 9 with traces of several other very minor complexes. This mixture was purified by preparatory thin layed chromatography using a $20 \times 20 \mathrm{~cm}, 500 \mu \mathrm{M}$ silica plate with ethyl actate as the mobile phase. $8(0.0085 \mathrm{~g})$ was isolated from a band $\left(\mathrm{R}_{\mathrm{f}}=0.49\right)$ that contained a $40 \%$ paramagnetic impurity by electrochemistry. ${ }^{1} \mathrm{H}$ NMR $\left(\mathrm{CD}_{3} \mathrm{CN}\right): \delta 1.19\left(\mathrm{~d}, 9 \mathrm{H}, \mathrm{J}=8.7 \mathrm{~Hz}, \mathrm{PMe}_{3}\right), 1.75(\mathrm{~d}, 1 \mathrm{H}, \mathrm{J}=9.9$ $\mathrm{Hz}$ ), 2.59 (d, 1H, J=16.8 Hz), 3.08 (dd, 1H, J=16.8, 9.0 Hz), 3.47 (dd, 1H, J=9.0, 4.2 Hz), $3.53(\mathrm{dd}, 1 \mathrm{H}, \mathrm{J}=12.9,9.9 \mathrm{~Hz}), 3.76(\mathrm{~s}, 3 \mathrm{H}), 3.82(\mathrm{~s}, 3 \mathrm{H}), 3.92(\mathrm{~d}, 1 \mathrm{H}, \mathrm{J}=4.2), 6.18(\mathrm{t}, 1 \mathrm{H}$, $\mathrm{J}=2.4 \mathrm{~Hz}, \mathrm{Tp}), 6.31$ (t, 1H, J=2.4 Hz, Tp), 6.45 (t, 1H, J=2.4 Hz, Tp), 7.44 (d, 1H, J=2.4 Hz, Tp), 7.46 (d, 1H, J=2.4 Hz, Tp), 7.73 (d, 1H, J=2.4 Hz, Tp), 7.88 (d, 1H, J=2.4 Hz, $\mathrm{Tp}), 7.93$ (d, 1H, J=2.4 Hz, Tp), 8.17 (d, 1H, J=2.4 Hz, Tp). ${ }^{13} \mathrm{C} \mathrm{NMR}\left(\mathrm{CDCl}_{3}\right): \delta 13.0(\mathrm{~d}$, $\mathrm{J}=29 \mathrm{~Hz}), 31.2(\mathrm{~s}), 36.3$ (s), 41.9 (s), 42.5 (s), 52.0 (s), 52.2 (s), 60.8 (s), 62.3 (d, J=12 Hz), 106.3 (s), 106.6 (s), 107.3 (s), 136.1 (s), 136.8 (s), 137.0 (s), 140.1 (s), 143.0 (s), 143.5 (s), 145.2 (s), 149.6 (s), 161.7 (s), 162.4 (s), 208.0 (s). ${ }^{31} \mathrm{P}$ NMR (CD $\left.{ }_{3} \mathrm{CN}\right): \delta-8.0$. IR: $v_{\mathrm{NO}}=1567, v_{\mathrm{CO}}=1618, v_{\mathrm{CO}}=1713 \mathrm{~cm}^{-1} . \mathrm{CV}: \mathrm{E}_{\mathrm{p}, \mathrm{a}}=+1.03 \mathrm{~V} . \mathrm{Ms}(\mathrm{ESI}):[\mathrm{MH}+]=$ 740. 
Spectra for compounds 6 and 8 .

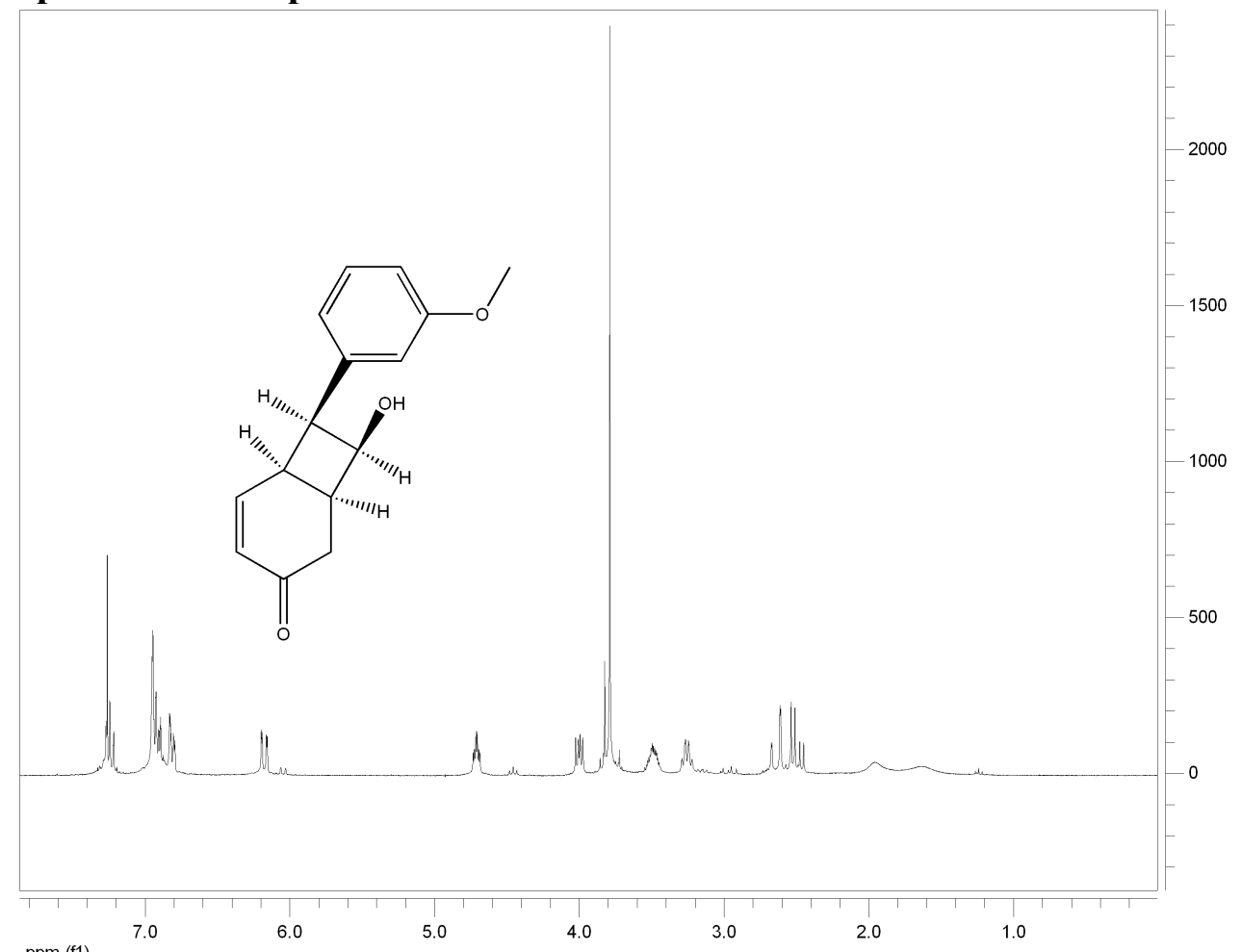

Compound 6 HNMR spectrum.

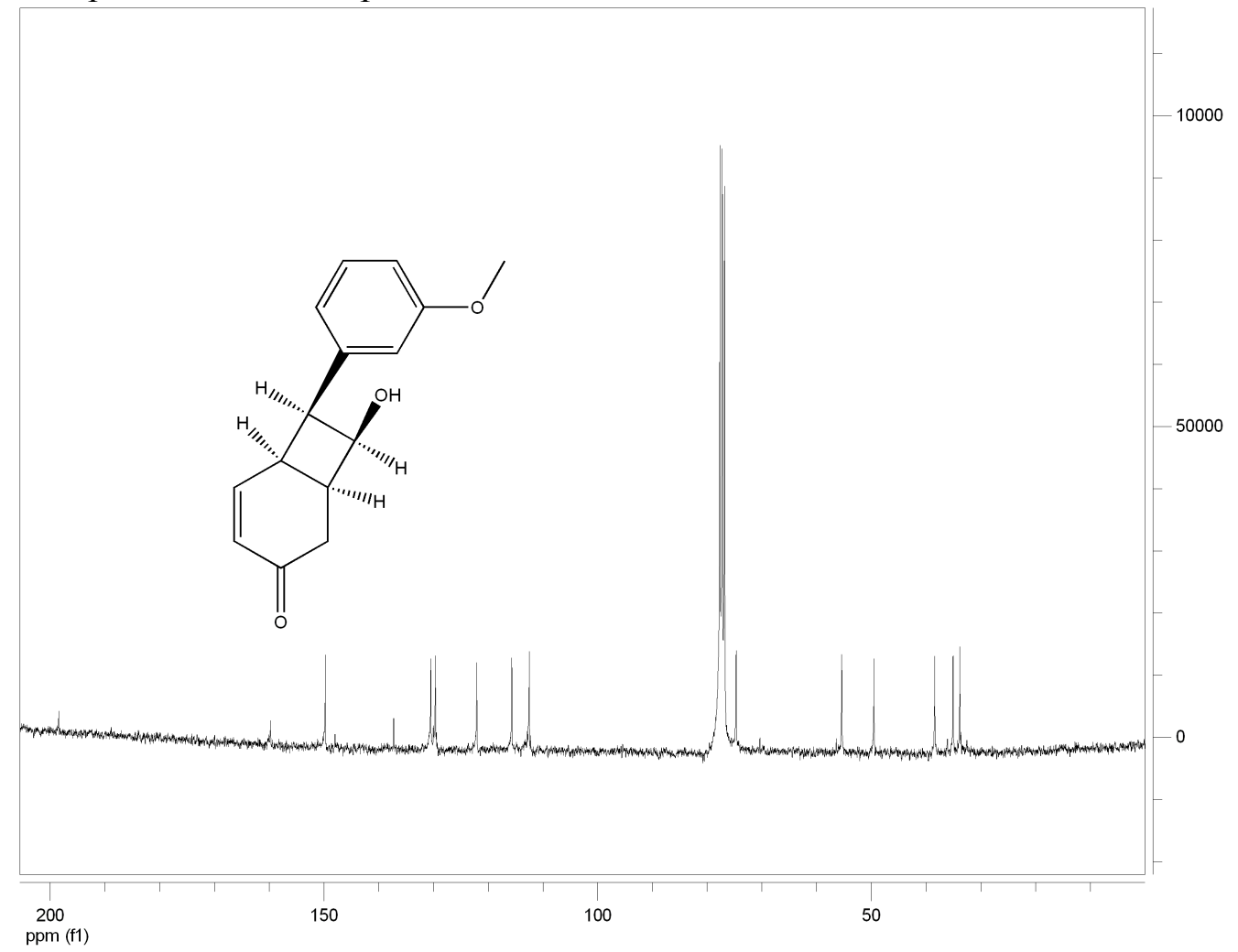

Page S7 
Compound $6{ }^{13} \mathrm{C}$ NMR spectrum.

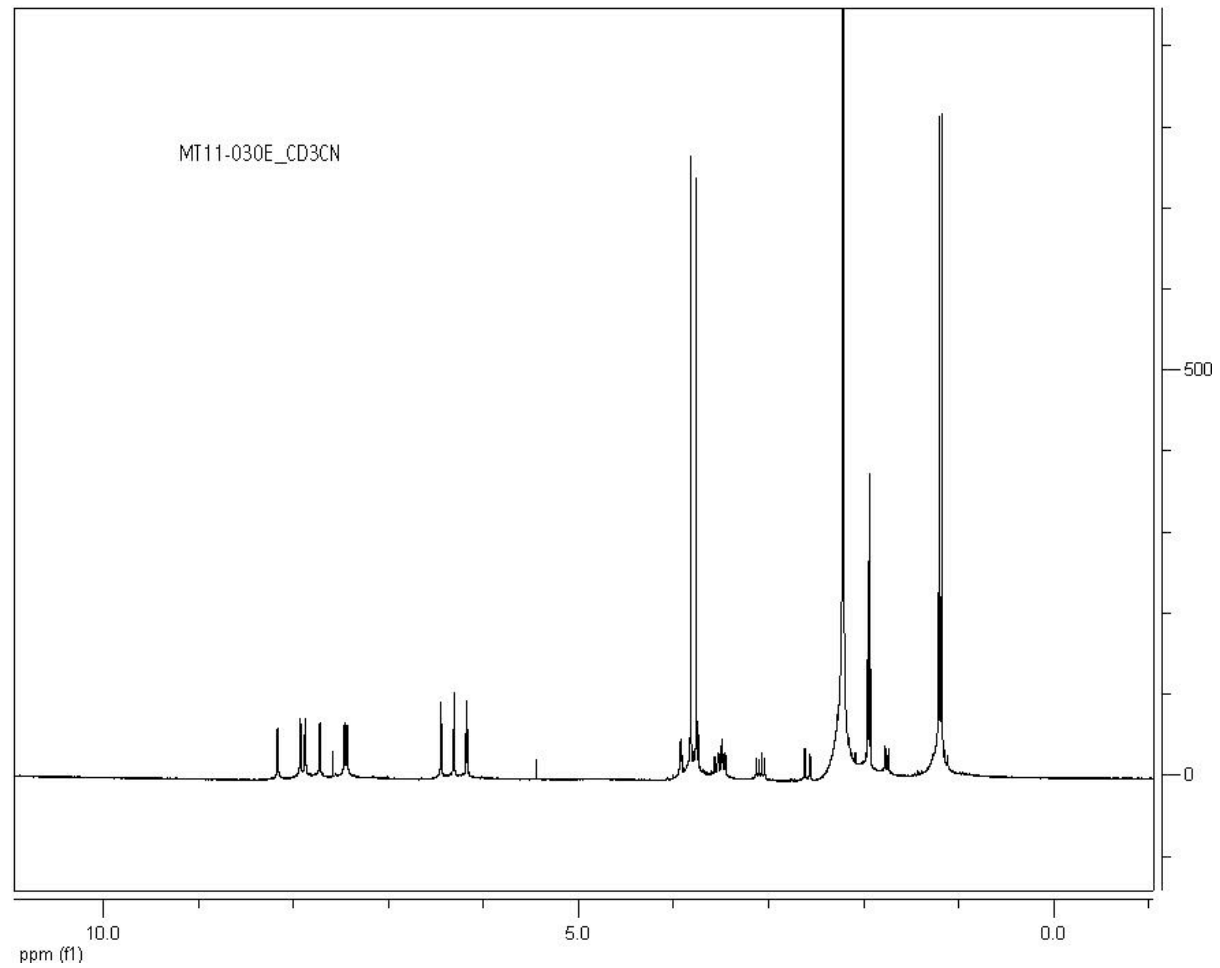

Compound 8 HNMR spectrum.

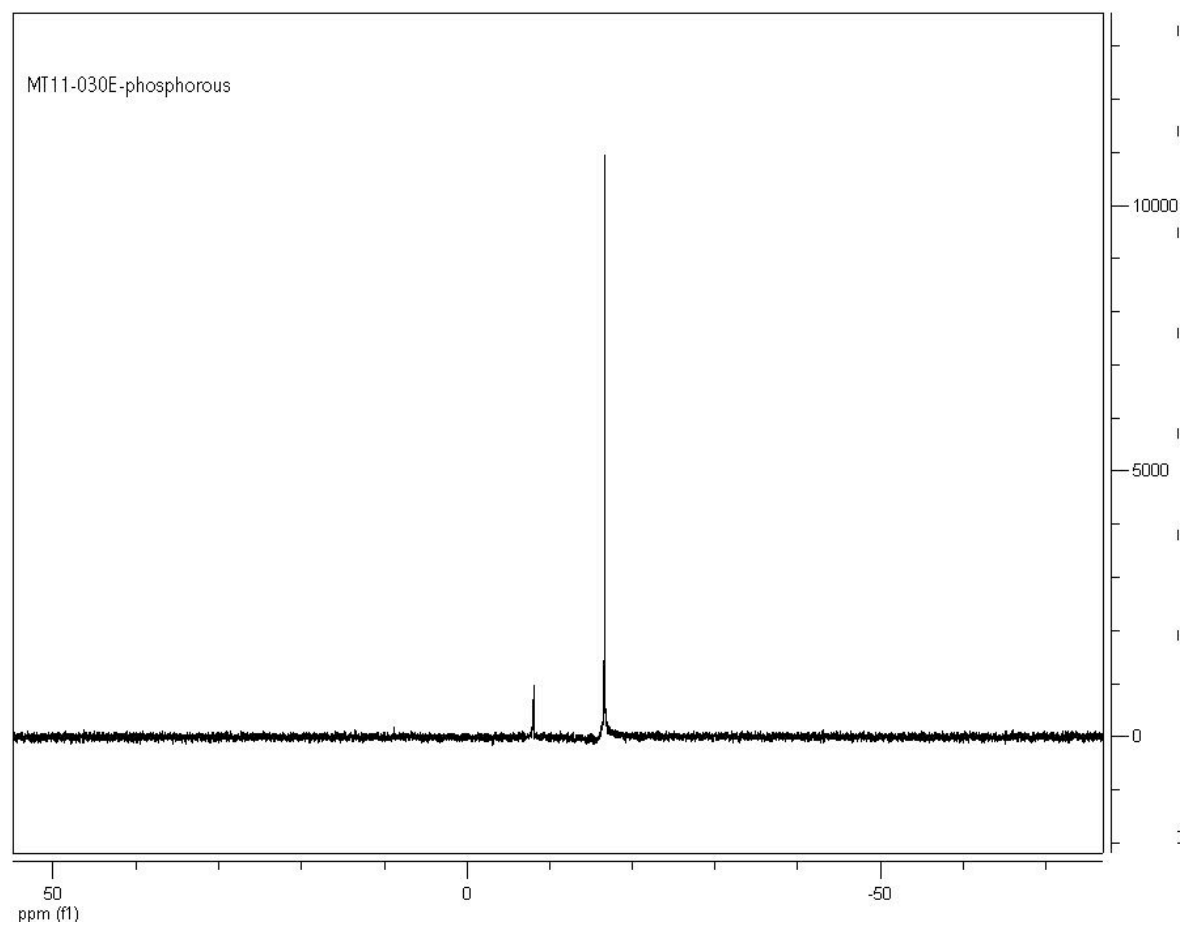

Compound $8{ }^{31} \mathrm{P}$ NMR spectrum. 


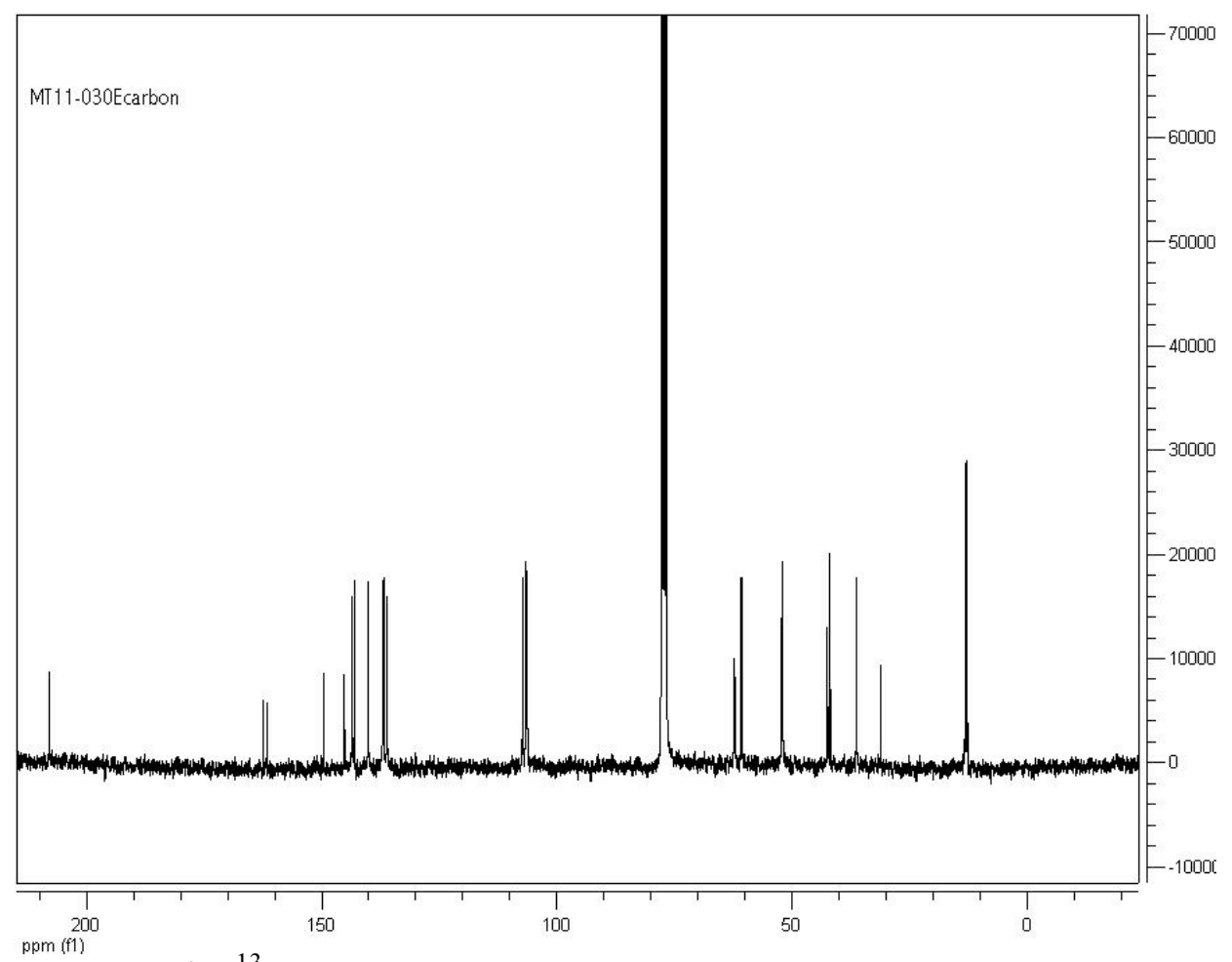

Compound $8{ }^{13} \mathrm{C}$ NMR spectrum. 